\title{
The Fingers as Sites of Leprosy Bacilli in Pre-relapse Patients
}

\author{
W. H. JOPLING, R. J. W. REES, D. S. RIDLEY*, \\ M. J. RIDLEY AND N. M. SAMUEL
}

The Hospital for Tropical Diseases, London NWI OPE, the National Institute for Medical Research, London NW7 and the Middlesex Hos pital Medical School, London W1

\begin{abstract}
Two dapsone treated patients with apparently quiescent lepromatous leprosy were found to have solid-staining acid-fast bacilli (AFB) in the fingers. The viability of the bacilli from both patients was proved and dapsone resistance was established in one patient by mouse foot-pad inoculation. Subsequently both patients relapsed bacteriologically, one clinically in addition. Thus solid-staining AFB in the fingers, though there may be none seen in skin lesions, may be the prelude to relapse.

It is suggested that the dorsum of fingers is a favourable site for persister bacilli because it is cool and nerve bundles are more superficial there than in most other areas.
\end{abstract}

\section{INTRODUCTION}

It has been found that acid-fast bacilli (AFB), sometimes solid-staining, may be found in slit-skin smears from the dorsum of the fingers of long-treated patients with lepromatous leprosy when other sites are bacteriologically negative. This was the more striking in that there were no lesions on the fingers (Ridley et al., 1976). These results were confirmed by Hiramalini et al. (1978), who obtained similar results also from the dorsum of the toes of patients with open footwear. Follow-up studies were not performed.

The purpose of the present paper is to report two cases of relapse, confirmed by mouse foot-pad inoculation, in patients in whom solid-staining AFB had previously been detected in fingers.

\section{Case Reports}

CASE 1

Mr J. T. is a Nepalese who was examined in his home country in May 1973, at the age of 21 , and was found to have 5 skin lesions which were insensitive.

* Requests for reprints at Hospital for Tropical Diseases. 
There is no record of nerve examination at this time. Skin smears were negative for AFB and a skin biopsy was reported as borderline leprosy.

\section{Progress}

Treatment with dapsone was instituted and was continued (with a break of 2 months) until he was seen in London in February 1976, when a clinical diagnosis of borderline-tuberculoid (BT) leprosy was made on the strength of a moderate number of purplish skin lesions, asymmetrically situated, all flat (macular), with a fairly dry surface, and all showing some degree of sensory impairment. Eyebrows and ears appeared normal. Several nerve trunks were thickened, and there was anaesthesia over the right forearm and over both legs and feet. The first intimation of a wrong clinical classification came from skin smears; these showed a BI of $3.5+$ and an SFG index of 2.0. It is noteworthy that the only solid-staining bacilli were found in the normal-looking skin on the dorsum of fingers. Histology of two skin lesions was that of subpolar lepromatous leprosy in regression. Mitsuda reaction was negative.

Treatment with dapsone was continued, and 2 months later he underwent a type 2 lepra reaction with erythema nodosum leprosum (ENL) and nerve pain, recurring over the next 3 months and requiring treatment with prednisone; there was one bout of left-sided epididymo-orchitis. By the end of July 1976 all reaction had ceased, his skin lesions appeared the same as they had done 5 months previously, and there were no new lesions. Skin smears were as follows:

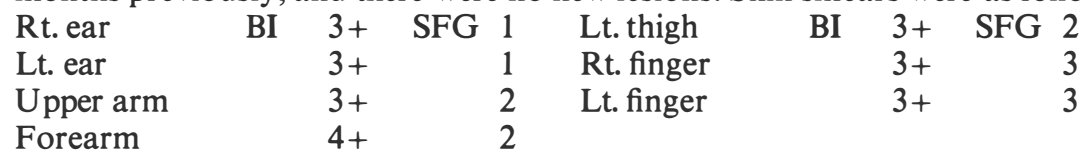

Thus although there were no lesions on the fingers (unlike the arm, forearm and thigh), bacilli in smears from fingers were as numerous as those in smears from these lesions. Once again fingers were the only source of solid-staining AFB (SFG 3).

At this time a biopsy of skin was taken from the dorsum of one of the middle fingers. This specimen weighed $0.068 \mathrm{~g}$ and yielded a total of $7.3 \times 10^{4}$ AFB, of which $2.5 \times 10^{3}$ were inoculated into both hind footpads of 6 thymectomized-irradiated mice. Bacilli were too few for an adequate $\mathrm{MI}$, but the majority were degenerate. However, 3 of 8 footpads harvested 8 to 9 months later showed significant multiplication (28 to 40 fold increase), confirming the presence of small numbers of viable bacilli in the fingers.

The patient was re-examined in January 1977. Although his skin lesions appeared to be unaltered and there was no sign of reaction, the skin smears showed deterioration, with solid-staining AFB now present in ear lobes. The evidence of relapse at this stage was considered to warrant a change of treatment, but progress could not be followed owing to the patient's return to Nepal.

CASE 2

Mr S. S. came to England as a Pakistani student in 1957. When he was first seen in London in October 1961, aged 24, he stated that for about 5 months he 
had experienced nasal symptoms consisting of blocked airway and occasional epistaxis and for about 3 months he had noticed papules on face and ears. On examination there were many skin-coloured papules on face, mostly on chin and ears, and both ear lobes were thickened. Eyebrows appeared normal. Several nerve trunks were thickened. Skin smears showed large numbers of AFB, mostly solid-staining. The histology of a papule was that of active subpolar lepromatous leprosy.

\section{Progress}

He was treated with dapsone and all skin lesions slowly disappeared. By October 1969, 8 years after commencing treatment, he had the first set of completely negative smears. His skin continued to remain normal, and smears to remain negative, until 1976 (nearly 15 years after beginning treatment), when his fingers were examined for bacilli in the course of the research study to which reference has already been made. These smears gave a BI of $3+$ and some solid-staining bacilli were present, although smears from ear lobes were negative and there were no skin lesions. Six months later (January, 1977), while continuing dapsone therapy, his skin was still clear of lesions but smears taken at random gave results as follows:

$\begin{array}{lrl}\text { ear } & \text { BI } 1+\text { (granular) } \\ \text { right upper arm } & 1+\text { (some solids) } \\ \text { left upper arm } & 3+\text { (some solids } \\ \text { two fingers } & 4+\text { (some solids) }\end{array}$

At this time a biopsy of skin was taken from the dorsum of one of the middle fingers. This specimen weighed $0.037 \mathrm{~g}$ and yielded a total of $4.1 \times 10^{5}$ AFB, of which $1.2 \times 10^{4}$ were inoculated into both hind footpads of 3 thymectomised-irradiated mice. All footpads harvested 8 months later showed significant multiplication (130 to 750 fold increases), confirming the presence of viable bacilli in the fingers. Bacilli harvested from these mice were passaged to further groups of untreated and dapsone treated mice, to determine their dapsone sensitivity. The bacilli multiplied in the groups of mice fed levels of $0.0001 \%$ and $0.001 \%$ dapsone, thus indicating a significant but intermediate degree of dapsone resistance.

After a further 6 months, 1 year after the finding of bacilli in fingers, macules typical of borderline leprosy were found on his back and around the waist. They looked slightly erythematous and active, but not in reaction. A biopsy of skin from one of these lesions showed an active BL lesion, which indicated that some upgrading had occurred during the period of remission. Bacilli were most conspicuous in a Meissner corpuscle.

Scanty AFB were present in nasal secretions, singly and in globi (but insufficient for animal inoculation). Evidence of relapse was conclusive. The patient responded well to a change of treatment.

\section{Discussion}

Of the two patients presented here, one had become clinically quiescent and the second apparently cured as a result of dapsone therapy, when solid- 
staining AFB were discovered as a result of examining smears from the dorsum of a finger. In each case the number of viable bacilli obtained on biopsy was sufficient to infect mice, and in the second case the bacilli were proved to be resistant to dapsone at two of the three dapsone levels at which sensitivity testing was carried out. There is some suggestion of dapsone resistance in the first case also, since there was evidence that the patient downgraded from BT to subpolar LL leprosy while on dapsone therapy. In both cases these findings were followed by a relapse of the infection with the reappearance of solid-staining bacilli at other sites and, in one case, of new skin lesions. It is apparent, therefore, that the dorsum of the fingers (or toes) is the site which produces the highest BI and the greatest chance of obtaining solid-staining bacilli (Ridley et al., 1976; Hiramlini et al., 1978); and also that the solid-staining bacilli so discovered may predict a clinical and bacteriological relapse.

In this study the skin smears were taken from the dorsum of the first phalanx. Hiramalini et al. (1978) found more solid-staining bacilli in the dorsum of the terminal than the middle phalanx. The pulp and palmar aspects of the fingers have not been investigated as sites of bacilli, because they are inconvenient sites for the patient for the taking of smears. However, it seems likely that the reason why the fingers are a site of predilection for persister bacilli, drug resistant or otherwise, is that they are a cool site, and also that on the dorsal surface the relatively large deep nerve bundles are more superficial than elsewhere because of the thinness of the dermis in this position. This would not apply so much to the palmar surface where the dermis is thicker. This hypothesis is supported by some unpublished observations on finger biopsies. The nerve bundles on the dorsum were convoluted, and AFB were present in the nerve bundles and sometimes in perineurial lepromatous granulomata. Expanded nerve endings (Meissner corpuscles only) were not conspicuous and they were not much involved by AFB. Thus the preponderance of these nerve endings is not likely to be the explanation for the predilection of leprosy bacilli for the fingers, as was suggested in the earlier study (Ridley et al., 1976).

\section{Ref erences}

Hiramalini, S., Joseph, N. A. and Chacko, C J. G. (1978). Concentration and persistence of bacilli in the fingers and toes of patients with lepromatous leprosy. Lepr. Rev. 49, 223.

Ridley, M. J., Jopling, W. H. and Ridley, D. S. (1976). Acid-fast bacilli in the fingers of long treated lepromatous patients. Lepr. Rev. 47, 93. 